\section{An improved inverse method for multirow blades of turbomachinery}

\author{
Li Aiting ${ }^{1,2} \oplus$, Zhu Yangli ${ }^{1,2,3}$, Li Wen ${ }^{1,2,3}$, Wang Xing ${ }^{1,2,3} \oplus$, \\ Qin $\mathrm{Wei}^{2,4}$ and Chen Haisheng ${ }^{1,2,3}$ (1)
}

Proc IMechE Part C:

J Mechanical Engineering Science

$0(0) \mathrm{I}-\mathrm{II}$

(C) IMechE 2020

Article reuse guidelines:

sagepub.com/journals-permissions DOI: $10.1177 / 095440622092445$

journals.sagepub.com/home/pic

\begin{abstract}
A three-dimensional viscous inverse design method is improved and extended to multirow blades environment. The inverse method takes load distribution as optimization objective and is implemented into the time-marching finitevolume Reynolds-averaged Navier-Stokes solver. The camber line of rotor blade is updated by virtual displacement, which is calculated by characteristic compatibility relations according to the difference between target and actual load so as to control the location and intensity of shock wave, and realize the optimization of flow structure and reduction flow separation. The inlet and outlet geometry angles of stator blade are adjusted in real time according to the inlet and outlet flow angles. Thus, it is computationally ensured that the blade row interactions are accounted and optimization process is carried out under the design condition. To preserve the robustness of calculation, the maximum virtual displacement is limited by $\mathrm{Y}+<\mathrm{I} 0$ and the camber line is smoothed via cubic B-spline interpolation. The complete blade profile is then generated by adding the prescribed blade thickness distribution to the camber line. The effectiveness of the method is demonstrated in the optimization of Stage 35 compressor stage. Numerical results showed that this inverse method can effectively improve the internal flow structure and optimize the matching between blade rows, and this method is robust, efficient, and flexible.
\end{abstract}

\title{
Keywords
}

Inverse design method, multirow blades, turbomachinery, load distribution, transonic compressor

Date received: 2 December 2019; accepted: 12 April 2020

\section{Introduction}

With the continuous development of computer performance, computational fluid dynamics (CFD) technology plays an increasingly important role in blade design and flow field analysis of turbomachinery. At present, most designers still adopt the direct approach, in which the designer first carries out onedimensional and two-dimensional design to obtain initial blade profile, then determine whether it meets the requirements with three-dimensional CFD calculation; if not, the blade profile is repeatedly modified according to the relevant experience of the designers until it meets the requirements. Due to the complexity of turbomachinery internal flow and the increase design index for advanced aero-engine compressor, the traditional design method often requires a lot of design time and cost, and relies heavily on experience and theoretical knowledge of designers. In this situation, the inverse method which determines a blade shape by a specific aerodynamic target can be a better choice since it allows direct control over local aerodynamic parameters which is required.
The early inverse design method is based on potential and stream function, which is generally limited to inviscid, incompressible and irrotational flows. ${ }^{1}$ The method was first applied in the design of two-dimensional blades. The three-dimensional method developed by Hawthorne et al. $^{2}$ designed blades from a specified average mean swirl (radius multiplied by tangential velocity). Later, the method was extended to transonic flows and some viscous effects were included. ${ }^{3,4}$ However, the methods were implemented under many assumptions and simplified conditions,

\footnotetext{
'Institute of Engineering Thermophysics, Chinese Academy of Sciences, Beijing, China

${ }^{2}$ University of Chinese Academy of Sciences, Beijing, China

${ }^{3}$ Dalian National Laboratory for Clean Energy, Dalian, China

${ }^{4}$ Institute of Mechanics, Chinese Academy of Sciences, Beijing, China
}

\section{Corresponding author:}

Zhu Yangli, Institute of Engineering Thermophysics, Chinese Academy of Sciences, West Road I I\#, North 4th Ring, Haidian District, Beijing 100190, China.

Email: zhuyangli@iet.cn 
and still had great limitations in practical application. Modern inverse methods, which is based on Euler and Navier-Stokes equation, breaks through the limitation of potential equation and can be used in threedimensional compressible rotational flow. There are mainly two kinds of inverse design methods that make full use of CFD technique. One is based on transpiration boundary conditions, which uses tangent condition of fluid on the blade surface to generate the blade profiles. This approach was first proposed by Léonard and Van Den Braembussche ${ }^{5}$ and later extended to three-dimensional applications by Demeulenaere and Van Den Braembussche. ${ }^{6}$ Dang's $^{7-9}$ group also developed a similar approach which employed a camber line generation equation constructing by tangent conditions to modify blade profile iteratively. The method presents higher calculation speed and robustness. However, due to the limited consideration of viscous effect by using slip boundary condition, the application in viscous flow field is limited. Another representative method utilized a virtual displacement, which was calculated from the difference between the current and target pressure on the blade surfaces to adjust the blade profiles. The method was first proposed by Thompkins and Tong ${ }^{10}$ and applied to the design of a two-dimensional blade profile. The convergence of this method is slightly poor, but it is easy to use in combination with most modern solvers and different grid structures; moreover, the selection of blade boundary conditions and turbulence model is not limited. Daneshkhah and Ghaly, ${ }^{11}$ Page et al., ${ }^{12}$ Arbabi et al., ${ }^{13}$ Nasrazadani et al., ${ }^{14}$ Yang et al. ${ }^{15,16}$ improved and extended the method to the full three-dimensional viscous flow field and achieved good results.

In the design of multirow blades, the aero-matching between blade rows has always been a difficult problem. The rotor-stator interactions which increase the complexity of flow field is a three-dimensional phenomenon induced by the wakes and vortices from upstream blades. The interaction between rotor and stator cannot be simulated accurately by traditional two-dimensional through-flow method. So the blade revision process to achieve proper matching is implemented in the three-dimensional diagnosing process, evaluating the performance of the matching state and modifying it as a function of calculation results based on design experience. This approach can be very time consuming and the result of optimization is strongly dependent on the experience of designers. The inverse method, which allows the direct modification of geometries to obtain an imposed performance, is a promising and attractive method. However, the existing inverse problem design methods of turbomachinery are mainly applied to the optimization design of single-row blades. Few studies have been done to apply them to the design of multirow blades and multistage turbomachinery. In the existing literature, only Van Rooij et al. ${ }^{17,18}$ have systematically studied the blade rows matching problem via three-dimensional inverse design method. They developed a pressure loading manager, which can automatically adjust pressure loading distributions to adapt the interaction between the rotor and stator. They compared the optimization results of rotors redesigned in single-row environment and multirow environment, and found that the obtained rotor blade produces pressure-loading distributions closer to design object when multirow inverse method is employed.

The inverse method shows an efficient design approach compared with the direct design method; however, it has not been widely used in the industrial design process. ${ }^{15}$ The main problem is that the robustness of the inverse design methods still needs to be improved. Moreover, the functional relationship between the aerodynamic parameters and blade shape has not been fully investigated, ${ }^{19}$ especially in multirow blade environment. There are two key factors are needed to be considered when dealing with multirow matching problems. Firstly, the given design objectives must be physically achievable, and secondly, an effective blade modification strategy that lead to optimal aero-matching should be proposed.

In this paper, an improved inverse method with multirow blades optimization capability was developed and validated. In order to deal with the aeromatching of rotor and stator blades flexibly, different methods are used to optimize the stator and rotor blades. For rotor blades, the blade profile was updated by virtual displacement calculated by the characteristic compatibility relations. For stator blades, the blade inlet angle and outlet angle were first adjusted according to the variation of inlet and outlet flow angle. Secondly, the blade profile was updated with smoothed target load distribution using virtual displacement method. The effectiveness of this approach was demonstrated by an application of a highly loaded transonic compressor stage. By comparing the aerodynamic performance parameters of original and optimized compressor, the effectiveness of the multirow inverse design method was verified.

\section{Numerical method}

\section{The basic flow solver}

To implement the study, a program for the internal flow of turbomachinery was developed. The program can be run in either flow analysis mode or inverse design mode, both sharing the same core solver. The core CFD solver used in the program is based on the integral form of the three-dimensional Reynolds-averaged Navier-Stokes (RANS) equation, given by

$$
\frac{d}{d t} \int_{\Omega} \mathbf{W} \mathrm{d} \Omega+\int_{S} \mathbf{F}_{c} \cdot \mathbf{n} \mathrm{d} S=\int_{S} \mathbf{F}_{v} \cdot \mathbf{n} \mathrm{d} S+\int_{\Omega} \mathbf{Q} \mathrm{d} \Omega
$$


where $\mathbf{W}$ is the vector of flow variables, $\mathbf{F}_{c}$ is the convective flux vectors, $\mathbf{F}_{v}$ is the viscous flux vectors, $\mathbf{Q}$ is the source item, $\Omega$ is the control volume, and $\mathbf{n}$ is the outward unit vector of the control volume boundary $S$.

The solver is pressure-based and uses traditional control volume discretization along with a time-stepping scheme. The advection upstream splitting method $^{20}$ scheme is used to calculate the convection flux, and the monotone upstream-centered schemes for conservation laws interpolation is used to improve the accuracy, while the inviscid fluxes are calculated based on the central difference scheme. The implicit lower-upper symmetric Gauss-Seidel ${ }^{21}$ method is used for time-marching integration. To facilitate the data processing in rotating coordinates, the equation is discrete in cylindrical coordinates.

A one-equation Spalart-Allmaras ${ }^{22}$ model in finitevolume form is used for the turbulence model. The construction of Spalart-Allmaras model is based on dimensionless analysis and experience. It is powerful enough to predict 3D mixing layers, wakes and flow separation and yields fairly rapid convergence to steady state, so as to be a good candidate for high load compressor stage. ${ }^{23}$

The nonreflective boundary condition is adopted in the inlet and outlet. At inlet, total pressure, total temperature, and flow angles in circumferential and radial directions are specified. At outlet, static pressure is imposed at the $50 \%$ blade height, and radial pressure distribution is determined by the simple radial equilibrium equation. Nonslip and adiabatic boundary conditions are used on the wall. For multirow calculations, the mixing plane model is used and carried out under the condition of equal flux to ensure the conservation of mass, momentum, and energy. The mixing plane model was chosen for the ability to captured first-order axisymmetric blade row interactions and computational convenience. ${ }^{24}$

The accuracy verification of CFD solver was carried out on Stage 35 compressor stage. Stage 35 is a low aspect ratio transonic axial flow compressor. It was designed according to the aerodynamic parameters required by a typical high-pressure ratio compressor in aircraft engine. NASA has measured the internal flow field of the compressor in detail. ${ }^{25}$ Its basic design and geometric parameters are shown in Table 1. A structured H-type grid is used as shown in Figure 1. Tip clearance is considered by pinching thickness of blade and set 14 layers of grid. The grid independence, monotone convergence and order of convergence of the solution are analyzed. The number of grids were determined by considering the calculation speed and accuracy. There are totally 560,000 nodes used in rotor blade and 350,000 nodes used in stator blade. The $Y+$ on the wall is kept from 1 to 5 . It takes no more than two hours to get convergence results. Comparison between the experimental data and the calculated results are
Table I. Design parameters of Stage35 compressor stage.

\begin{tabular}{ll}
\hline Parameters & Value \\
\hline Number of rotor blades & 36 \\
Number of stator blades & 46 \\
Rotor blade aspect ratio & 1.19 \\
Stator blade aspect ratio & 1.26 \\
Design speed $(\mathrm{r} / \mathrm{min})$ & $17,188.7$ \\
Design mass flow $(\mathrm{kg} / \mathrm{s})$ & 20.8 \\
Tip velocity $(\mathrm{m} / \mathrm{s})$ & 454.46 \\
Tip clearance $(\mathrm{mm})$ & 0.408 \\
\hline
\end{tabular}

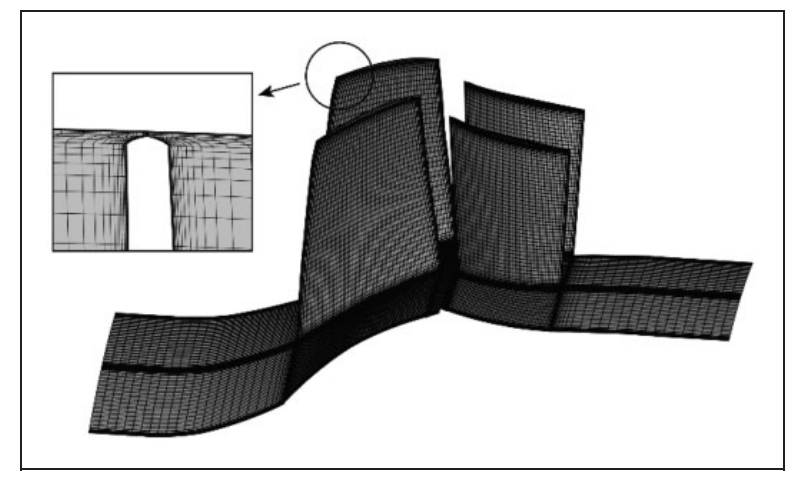

Figure I. Computational mesh used in the calculations.

shown in Figure 2. It showed good consistency in isentropic efficiency and total pressure ratio, which verified the accuracy of the developed solver.

\section{Inverse design methodology}

\section{Modification method of the rotor blade}

The key to inverse design method is the functional relationship between prescribed aerodynamic parameters and blade profiles. The current inverse method for rotor blade is based on an improved virtual displacement method. The relationship between aerodynamic parameters and blade profile is calculated by characteristic compatibility relations.

As shown in Figure 3, the static pressure on pressure surface and suction surface of the blade segment are $\mathrm{p}^{+}$and $\mathrm{p}^{-}$respectively, so the magnitude of the force on the blade is the pressure difference between the pressure and the suction surfaces, that is, the load on the blade which expressed in $\Delta p$. Assuming that the blade segment is a plate without thickness and the virtual movement can occur under the action of load, in order to obtain the relationship between virtual displacement and load, the following two simplifications are made for this physical process. First of all, the fluid on the blade surface only has the normal flow component under nonslip boundary condition, so the 3D flow model can be simplified as one-dimensional model. Secondly, the shear stress along the flow 


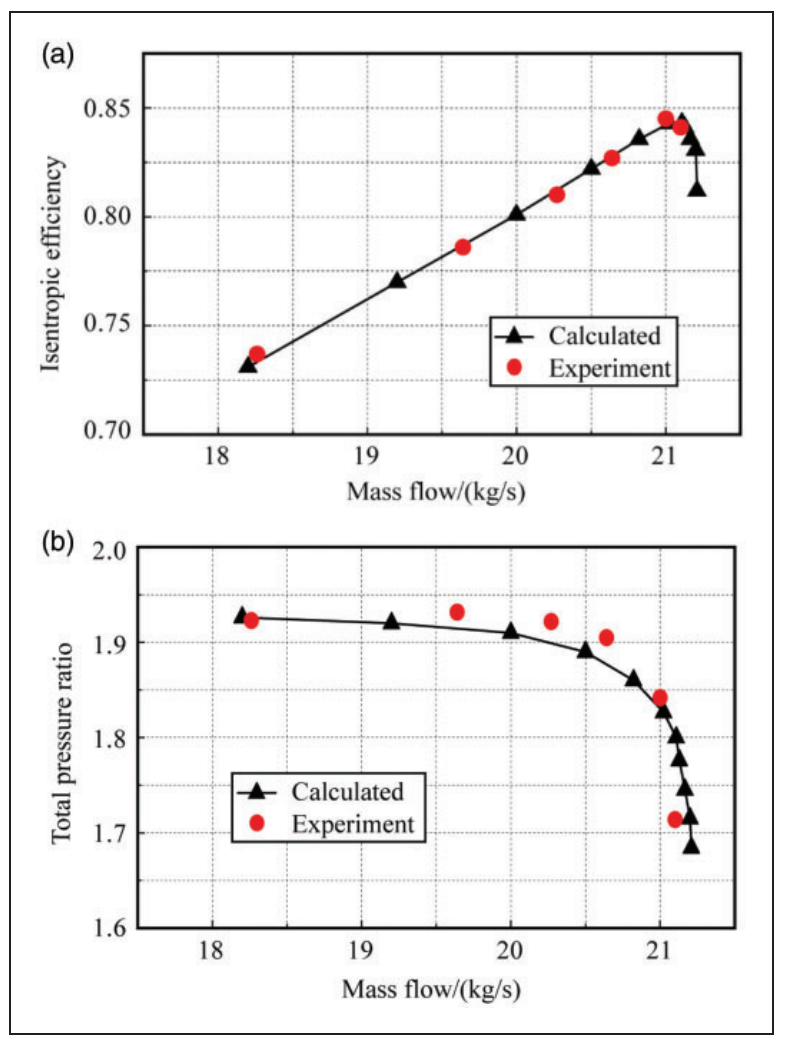

Figure 2. Comparison of the overall performance of Stage35 compressor stage: (a) isentropic efficiency; (b) total pressure ratio.

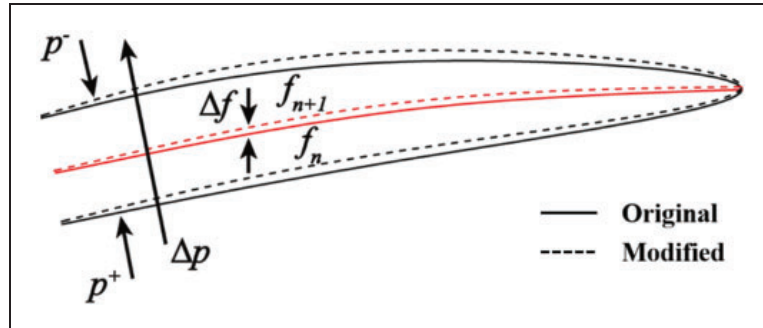

Figure 3. Blade profile virtual movement.

direction of fluid in the boundary layer is much greater than that in the normal direction, so the viscosity effect of the fluid can be ignored when calculating the normal movement of the fluid. In conclusion, the physical process is simplified as one-dimensional inviscid flow process. According to the theory of characteristics, the blade segment satisfies the following characteristic compatibility relations

$$
-\rho^{+} c^{+} \mathrm{d} V+\mathrm{d}(\Delta p)=0
$$

where $\rho^{+}$is the density of pressure surface, $c^{+}$is the sound velocity of pressure surface, $\mathbf{V}$ is the velocity of the fluid on the blade surface. By discretizing equation (3), we can get the following expression

$$
-\rho_{n}^{+} c_{n}^{+}\left(\mathbf{V}_{n+1}-\mathbf{V}_{n}\right)+\left(\Delta p_{n+1}-\Delta p_{n}\right)=0
$$

The subscripts $n$ and $(n+1)$ denote the time levels ( $n$ means the current one).

Assuming that no flow separation occurs, the velocity of the blade is equal to the fluid on the blade surface. Due to the nonslip boundary condition, the velocity of the current moment can be set to zero, which is $\mathbf{V}_{n}=0$. The load of the next moment can be set as the target load, which is $\Delta p_{n+1}=\Delta p_{\text {target }}$. Then the wall displacement formula of the blade is

$$
\Delta f=\varepsilon \Delta t \mathbf{V}_{n+1} \mathbf{n}_{\theta}=\varepsilon \Delta t \frac{\Delta p_{\text {target }}-\Delta p_{n}}{\rho_{n}^{+} c_{n}^{+}} \mathbf{n}_{\theta}
$$

where $\mathbf{n}_{\theta}$ represents the normal vector in the circumferential direction, $f$ represents the circumferential coordinate value of the camber line, $\Delta t$ is the physical time step of calculation, and $\varepsilon$ is a relaxation factor to control the stability of convergence.

The theory of characteristics is derived from the assumption of $1 \mathrm{D}$ inviscid fluid. So, the equation is applicable when the tangential velocity is very small. And we can see from equation (3) that the larger the difference between the target load and the actual load is, the larger the wall displacement will be; the larger the difference is, the more easily the blade will be distorted and the calculation will easily diverge. Therefore, in order to maintain the robustness of the calculation, the maximum wall displacement will be limited. $Y+$ can be used to judge the flow state of wall boundary layer. When $Y+$ is small, the viscosity of the fluid dominates, and the tangential velocity is small. So the maximum wall displacement can be limited by $Y+$, i.e.

$$
\Delta f_{\text {step }}=\min \left(\Delta f, \Delta f_{\text {max }}\right)
$$

where $\Delta f_{\text {step }}$ is the improved wall displacement, and the maximum value of virtual displacement $\Delta f_{\max }$ can be estimated by the following formula, ${ }^{26}$ which is used in NUMECA

$$
\Delta f_{\max }=6\left(\frac{V_{r e f}}{v}\right)^{-\frac{7}{8}}\left(\frac{L_{r e f}}{2}\right)^{\frac{1}{8}} Y^{+}
$$

where reference velocity $V_{\text {ref }}$ and reference length $L_{r e f}$ can be replaced by the average velocity of inlet and the chord length at the middle span of blade respectively. In our test, we find that the stability and convergence of the calculation are good when $Y+$ is less than 10. After the wall displacement is obtained, the circumferential coordinates of the camber line can be updated by the following formula

$$
f_{n+1}=f_{n}+\Delta f_{\text {step }}
$$

In order to maintain the stability of the iterative process, the smoothness of the blade profile should be guaranteed. Before generating the blade profile, cubic 
B-spline interpolation is used to smooth the blade camber line. As shown in Figure 4, where the aerodynamic parameters fluctuate greatly, the virtual displacement between adjacent grid points will deviate greatly, which will lead to the distortion of blade camber line. So, the modification of blade camber line is carried out on a series of selected control points, which can be distributed uniformly along chord or skipped where large load gradient exists, and the rest points are obtained by interpolation. After the blade camber line is updated, the prescribed blade thickness is added to the new camber line, and hence the complete blade profile is obtained.

\section{Modification method of stator blade}

The inlet and outlet flow angles of the blade is an important parameter for the matching design of the multirow compressor. The outlet flow angle of the rotor blade directly determines the inlet flow angle of the stator blade. At the design point of a compressor blade, the incidence close to zero degrees is preferable. In this case, the flow deflection in the passage is realized by the camber of the blades. When there is a positive incidence, the flow impinges on the pressure surface of blade, so the flow on the suction surface must accelerate rapidly near the leading edge. This results in very high local diffusion near the blade leading edge. The high local diffusion will lead to the transition of boundary layer, and the loss of blade is greater. At a very high incidence, the flow may separate and even lead to stall.

For high loaded transonic compressor stage, the flow field of rotor blade is complex, often accompanied by shock wave, transition, flow separation, and strong secondary flow. The method based on virtual displacement used in this paper can effectively control the shock intensity and position of the rotor blade, but it has limited effect on the modification of flow angle. The flow of stator blade is generally in subsonic state, and the degree of flow separation and secondary flow is weak. Therefore, the matching task of flow angle is completed by the stator blade.

When optimizing multirow blades by using inverse problem design method, the rotor blade profile varies according to the given load distribution, and the outlet flow angle of the rotor blade changes correspondingly, so the inlet flow angle of stator blade

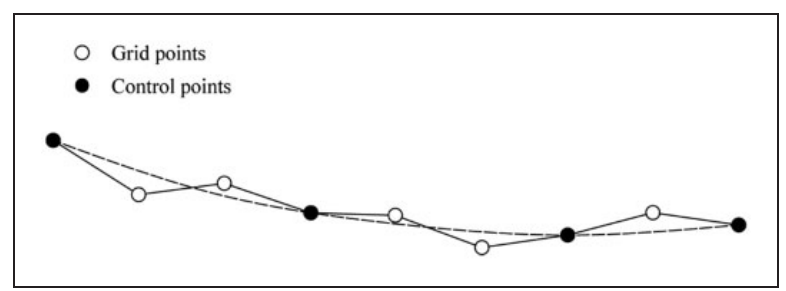

Figure 4. Cubic B-spline interpolation. varies accordingly. This has two main effects on the optimization results. Firstly, the variation of the flow angle increases the incidence, thus introducing additional mismatching losses. Secondly, the current working condition will deviate from the design condition due to the variation of incidence, so the adjustment of the rotor blade profile is carried out under off-design condition, which deviates from the designer's intention and may lead to the failure of convergence.

In order to reduce the interference of interaction between stator and rotor blade rows in the process of inverse optimization, the stator blade is adjusted with the variation of rotor blade profile. In more detail, for every iteration the rotor blade is corrected, the inlet flow angle of stator blade is recalculated, and the inlet blade geometry angle of stator blade is adjusted to match the inlet flow angle, i.e. the incidence is kept at 0 degrees during the inverse design process. For stator outlet, considering that the design of the next stage is based on the aerodynamic parameters of the stator outlet in order to reduce the bending and twisting degree of the next stage, it is necessary to ensure that the outlet flow angle distributes uniformly along spanwise. Therefore, for stator outlet, the uniform distribution of outlet flow angle along spanwise is taken as the adjustment principle. In each iteration, the average value of the outlet flow angle of the stator blade is calculated, and the outlet geometry angle of each section is adjusted according to the average value. Finally, in order to avoid the distortion of blade profile caused by the direct change of inlet and outlet blade geometry angle, the virtual displacement method is used to fine-adjust the stator blade profile after adjusting the inlet and outlet blade geometry angle. Different from the method used in rotor blades, the target load distribution is smoothed by cubic B-spline interpolation based on the current load distribution and changes dynamically with the iteration process.

When applying the inverse design method proposed in this paper, the solving steps are shown in Figure 5. Firstly, the initial blade profile data are used to generate grids, and the CFD solver is used to simulate the flow field. Secondly, the load distribution data of the rotor blade are extracted and compared with the target load distribution, and the virtual displacement is calculated to modify the blade profile. At the same time, the inlet and outlet flow angles of the stator blade are calculated according to the computed flow parameters. Then, the rotor blade profiles are modified according to the virtual displacement, the stator inlet geometry angle is adjusted according to the stator inlet flow angle, and the stator outlet geometry angle is adjusted according to the radial average value of the stator outlet flow angle. At last, the blade camber line is smoothed and the blade thickness distribution is added to generate the new blade profiles. If the calculated load does not conform to the 


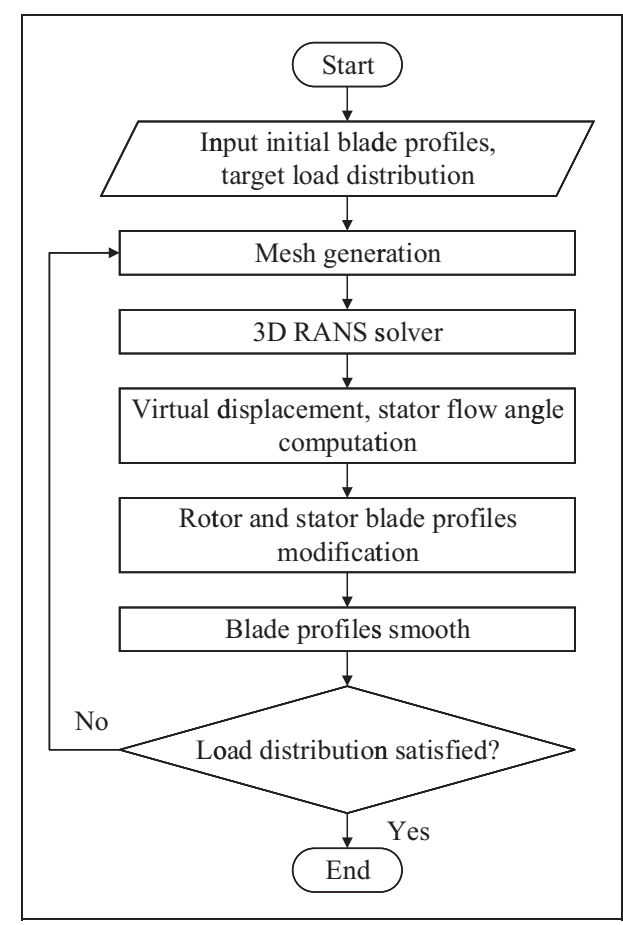

Figure 5. Flowchart of the inverse design method. RANS: Reynolds-averaged Navier-Stokes.

target load distribution, repeat the above process until the load distribution is satisfied.

\section{Inverse design implementation}

\section{Target load adjustment}

To verify the effectiveness of the proposed algorithm, the Stage35 compressor stage was selected for this verification. Stage 35 compressor is a typical transonic compressor rotor with small blade bend angle. Unlike subsonic compressor, it relies mainly on shock wave to increase pressure. The intensity and position of shock wave are related to the load distribution on the blade surface. Therefore, the shock wave and aerodynamic parameter distribution in the flow passage can be controlled by adjusting the load on the blade surface to improve the compressor performance.

In order to obtain a reasonable blade load adjustment strategy, the Stage35 compressor with the original blade geometry was analyzed at the design point. The contours of relative Mach number of the original blade profile are shown in Figure 6. It can be seen that the performance of the compressor is affected by the unexpected flow field distribution and three important conclusions can be obtained by inspection. (1) There is a bow shock at the leading edge of the blade, extending from the leading edge of the pressure surface to the middle of the suction surface, and intersecting with a channel shock at $Z=10 \%$ of chord, which forms a $\lambda$-shaped shock structure. This makes the aerodynamic parameters

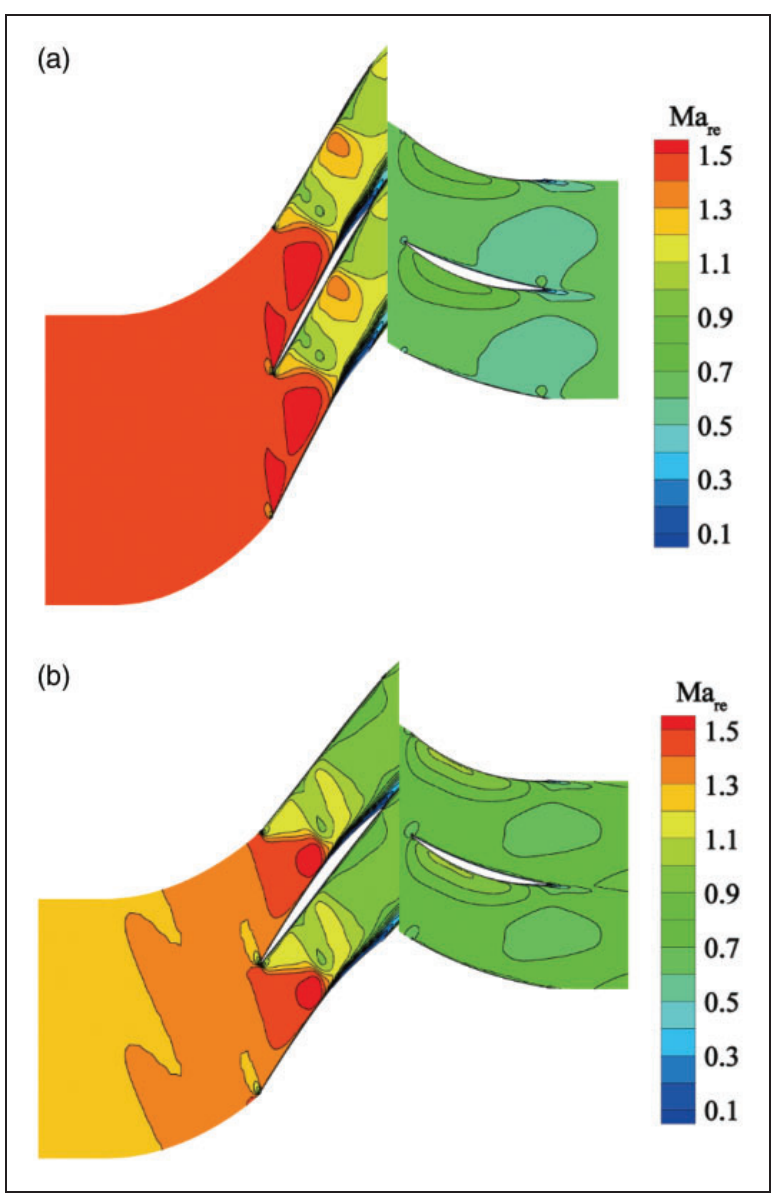

Figure 6. Relative Mach number contours of the original blade profile: (a) $R=90 \%$ span from hub; (b) $R=50 \%$ span from hub.

fluctuate sharply in this region, generates a vortex dissipation structure and introduces additional losses. (2) There is a region with high Mach number at $Z=50 \%$ of chord on suction surface, which is similar to the flow field structure of Laval nozzle and has a negative impact on the pressure rise. (3) In addition, a large flow separation zone can be observed at the suction side near trailing edge, which is caused by the large load on the blade trailing edge. Therefore, the performance of the rotor blade still has the potential to be improved.

The blade profile is modified in a section-by-section way. The inversely designed sections can be distributed uniformly along spanwise, or clustered where larger radial gradients exists. For Stage 35 compressor, nine uniform sections from $R=10 \%$ to $R=90 \%$ span are selected as calculation sections, and the load distribution of each section are adjusted respectively. Based on the above analysis, the specific revision principles are as follows. (1) Appropriately increasing the load and moving the position of the peak load to the leading edge of the blade, so as to improve the shock wave structure. (2) Reasonably smoothing the load distribution after shock wave to reduce the high gradient area of the load and make the load distribution 
uniformly. (3) Properly reducing the load near blade trailing edge to reduce flow separation phenomenon.

\section{Results and discussion}

According to the above three principles, the original load distribution on the blade surface was adjusted, and was taken as the target load distribution. The time consumed in the calculation of the inverse design process is almost the same as the time consumed in the convergence of the flow field. The modified load distribution is shown in Figure 7. It presents smoother variation, and the load decreases from leading to trailing edge. The peak load moves from $Z=10 \%$ of chord to the leading edge of the blade. Figure 7 compares the specified and inversely obtained load at different radial sections. It can be seen that the inversely obtained load distribution satisfies the specified target load well. It shows that the calculation results meet the design objectives and verify the stability and convergence of the method.

Figure 8 shows the contours of relative Mach number at $R=90 \%$ and $R=50 \%$ span of the modified blade. Compared with Figure 6, the flow structure in the blade passage was optimized according to the design intent. The $\lambda$-shaped shock wave near the blade leading edge is replaced by a normal shock wave. Compared with original blade, the Mach number at $Z=50 \%$ of chord on the suction surface decreases obviously, and more uniform distribution of aerodynamic parameters is obtained. The region with lower Mach number on the suction side of the trailing edge represents that the flow separation is significantly reduced. Figure 9 compares the static pressure distribution on the rotor blade at $R=90 \%$ and $R=50 \%$ span before and after optimization. It can be seen that the static pressure of the pressure side at the front of the blade increased and the static pressure of the suction side at the back of the blade decreased. Part of

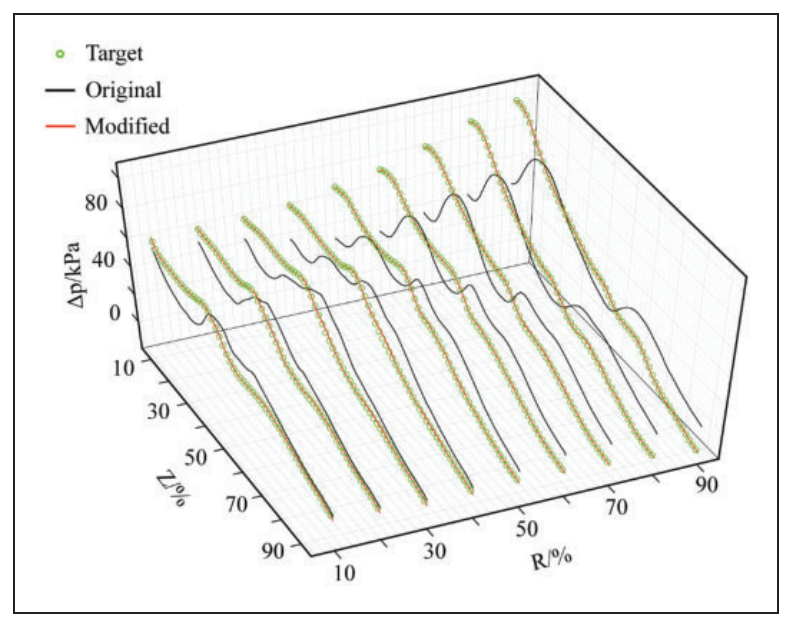

Figure 7. Comparison of original and modified 3D load distribution of rotor blade. the load acting on the back of blade is transferred to the front, which forms a forward load distribution. Above all, it can be seen that the internal flow structure of the blade is more reasonable after the optimization, and the expected goal of the design is achieved.

Figure 10 shows the spanwise distribution of the inlet blade geometry angle obtained from inverse design compared to the inlet flow angle and the original inlet blade geometry angle. It can be seen that the stator blade inlet geometry angle is in good agreement with the inlet flow angle. The deviation from the inlet flow angle is about $1^{\circ}$, which is due to the blade profile smoothing process. As a matter of experience, the positive incidence is beneficial to the off-design condition, so the small incidence was retained.

Figure 11 shows a comparison of the spanwise distribution of the outlet flow angle before and after optimization. It can be seen that the outlet flow angle obtained from inverse design method fluctuates less along the spanwise direction, indicating that the aerodynamic parameters are more uniform along spanwise. It is beneficial to the design of the next stage rotor blade. The degree of bending and twisting

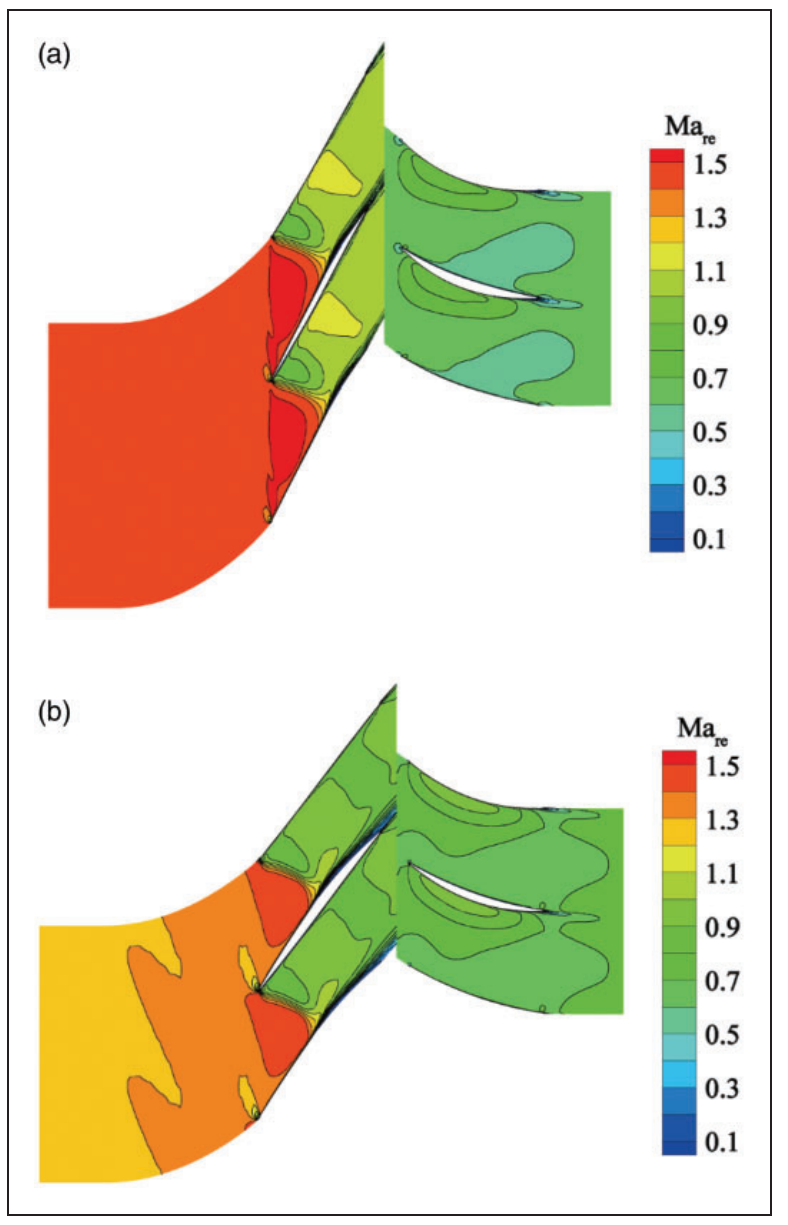

Figure 8. Relative Mach number contours of the optimized blade profile: (a) $R=90 \%$ span from hub; (b) $R=50 \%$ span from hub. 


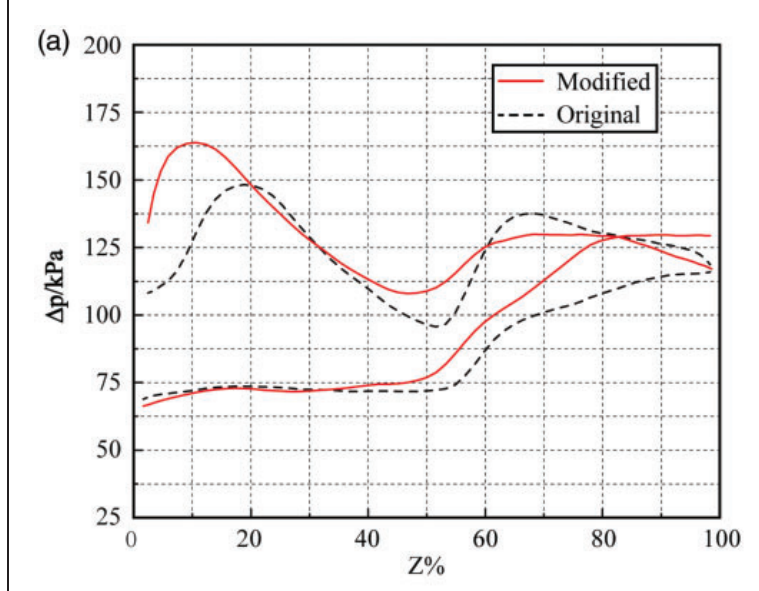

(b)

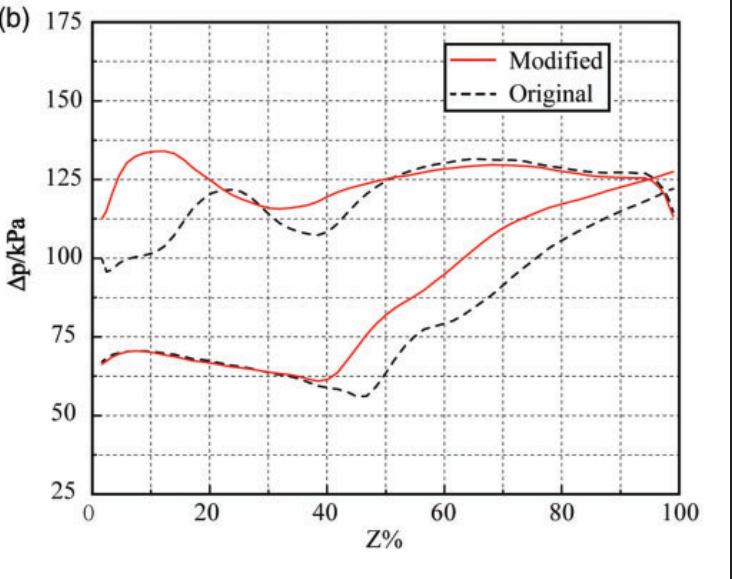

Figure 9. Comparison of the original and modified static pressure distribution of rotor blade: (a) $R=90 \%$ span from hub; (b) $R=50 \%$ span from hub.

of the next stage rotor blade can be reduced, thus reducing the difficulty of blade modeling.

To explain the necessity of fine adjustment of stator blade profile by virtual displacement method, Figure 12 compares the load distribution with and without adjustment at $R=90 \%$ span of stator blade. It can be seen that the load distribution without fine adjustment fluctuated to some extent, while the load distribution after fine adjustment tended to be smooth.

The overall performance of the redesigned blade under design point is shown in Table 2. The mass flow rate was basically unchanged, the isentropic efficiency of the compressor was increased by $1.2 \%$, and the total pressure ratio was slightly increased. Figure 13 shows a comparison of the off-design performance curves of the compressor. The isentropic efficiency of the optimized compressor stage was better than that of the prototype compressor in the whole working condition. The total pressure ratio was higher than that of the prototype compressor in high mass flow condition and was slightly lower in low mass flow condition, but overall, the performance of the compressor stage has been improved. Figure 14 shows a comparison of the blade shape before and

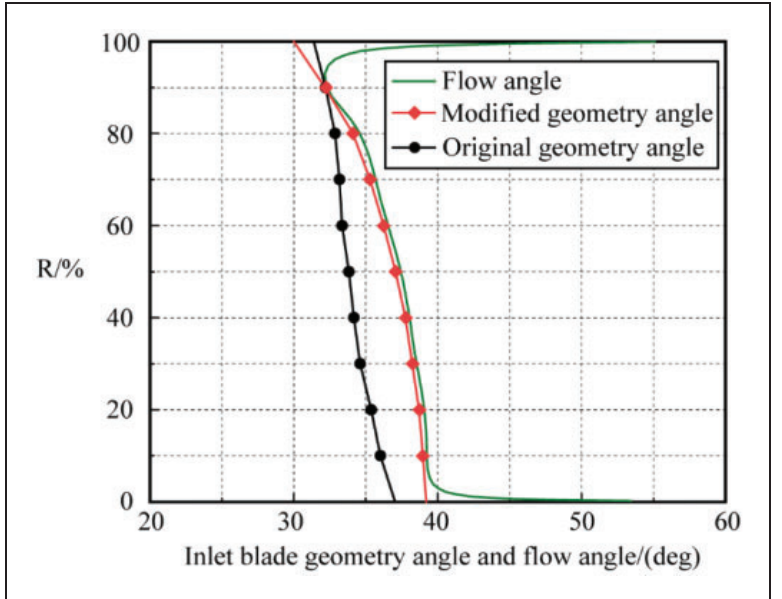

Figure 10. Comparison of original and modified inlet blade geometry angles of stator blade.

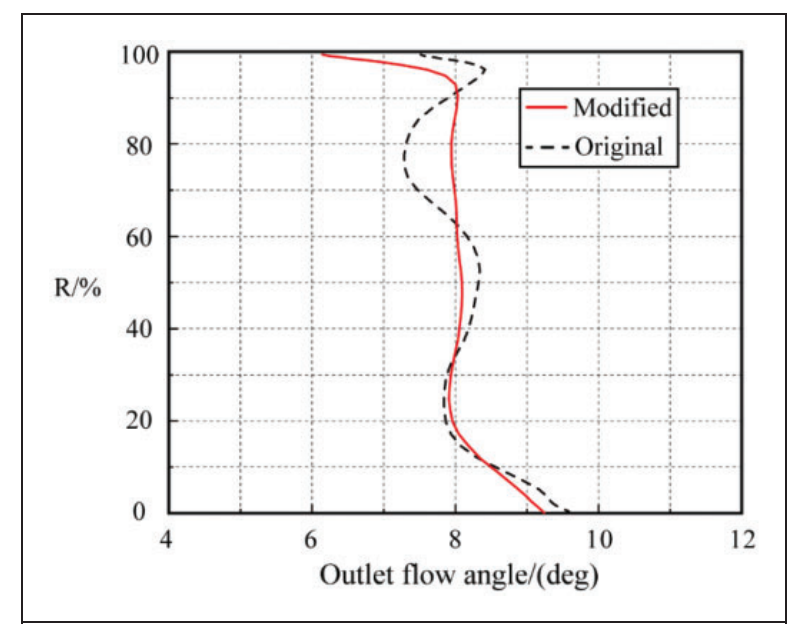

Figure II. Comparison of the original and modified outlet blade geometry angles of stator blade.

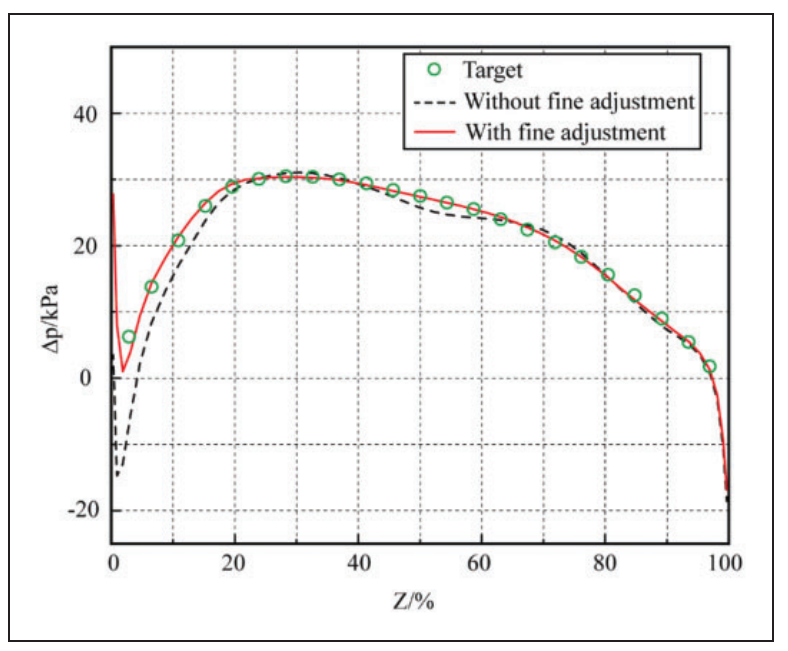

Figure 12. Comparison of the load distribution of stator blade with and without fine adjustment at $R=90 \%$ span. 
Table 2. Performance comparison of the original and modified blade profile at design point.

\begin{tabular}{lcc}
\hline Parameters & Original & Modified \\
\hline Mass flow rate $/\left(\mathrm{kg} \cdot \mathrm{s}^{-1}\right)$ & 21.02 & 21.04 \\
Total pressure ratio of stage & 1.826 & 1.844 \\
Isentropic efficiency of stage & 0.843 & 0.855 \\
\hline
\end{tabular}

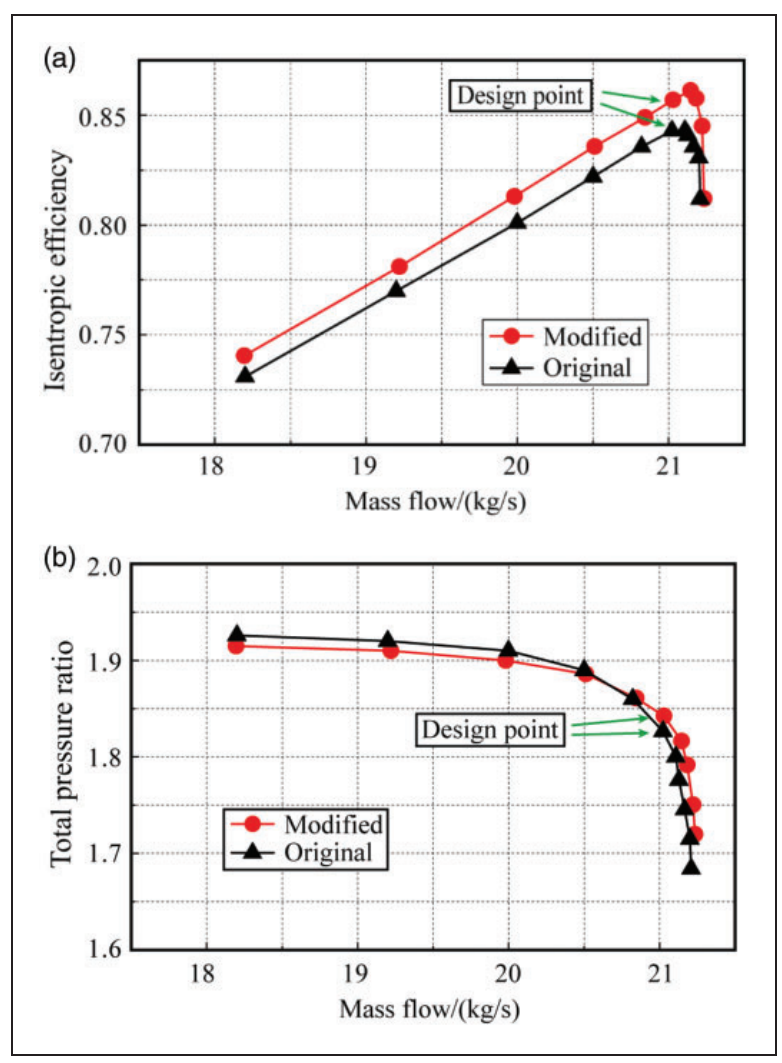

Figure 13. The overall performance comparison of the original and modified blade profile: (a) isentropic efficiency; (b) total pressure ratio.

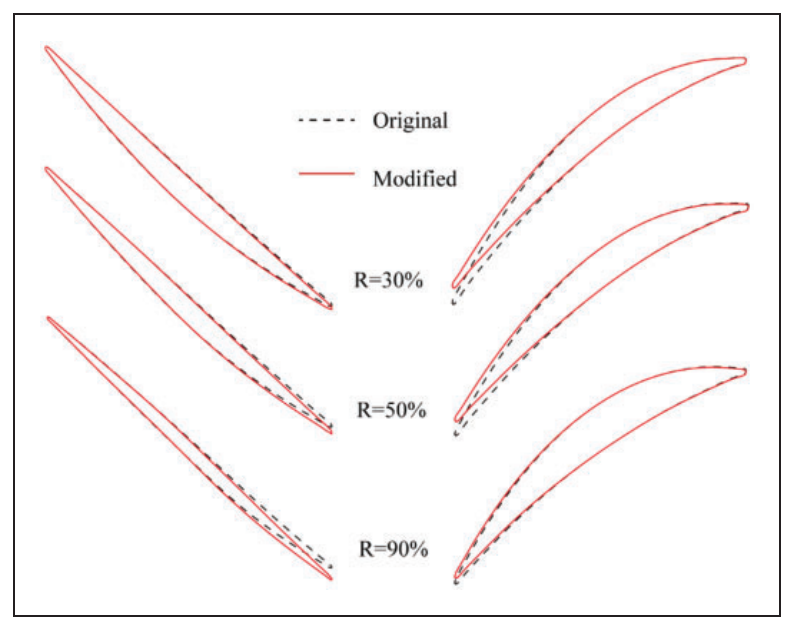

Figure 14. Comparison of the original and modified blade profile.

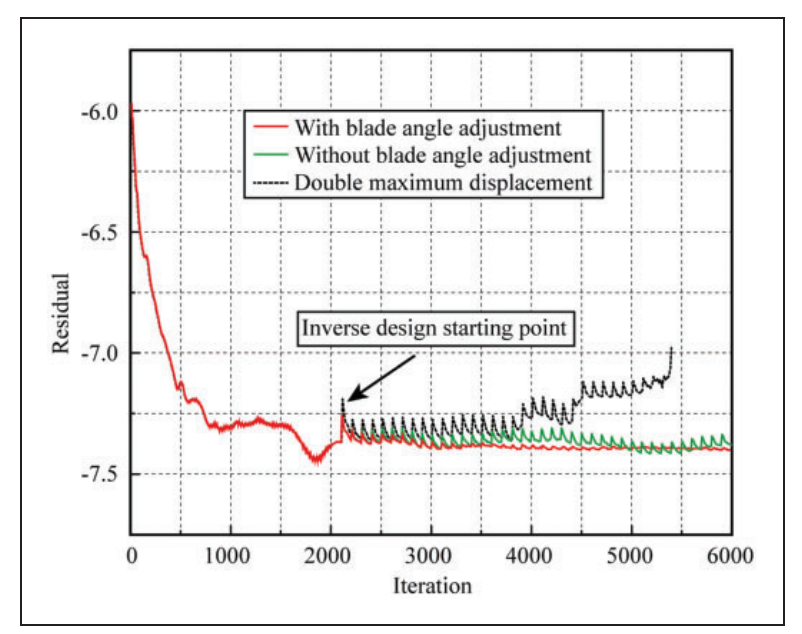

Figure 15. Comparison of residual curves under different treatments.

after optimization. Both rotor blade and stator blade have been slightly adjusted after optimization.

To demonstrate the advantages of the improved inverse design method, the two other cases were carried out for comparison. In one case, the geometry angle of stator blade was fixed. For the other case, the maximum virtual displacement was set to $Y+=20$. Figure 15 shows the comparison of the residual convergence curves of the three cases. It can be seen that when the blade geometry angle was fixed, its residual curve always fluctuated, indicating that the operation condition deviated from the design point and the target load distribution cannot be achieved. When the maximum virtual displacement was set to $Y+=20$, its residual curve fluctuated more violently, indicating that the modification of blade shape was more sensitive to the difference of load. As the number of iteration steps increased, the distortion degree of blade exceeded the limitation, thus leading to the breakdown of the solver.

\section{Conclusion}

In this paper, an improved full three-dimensional viscous inverse design method based on the virtual displacement is proposed and adopted in multirow blades environment. In this method, the virtual displacement is derived from the compatibility relationship and calculated according to the difference between the target load and the actual load. The maximum virtual displacement is limited by $Y+<10$. To enhance the aerodynamic matching between rotor and stator blade rows, the stator blade inlet and outlet angles is adjusted according to the inlet and outlet flow angles. The blade profile is smoothed by cubic B-spline interpolation.

The effectiveness and robustness of the improved method are demonstrated through stage 35 compressor stage and the following conclusions are obtained. The inverse design method developed in this paper 
can be applied to the multirow blades environment with good stability, convergence, and acceptable computational time consumption. The aerodynamic parameters distribution of the blade can be effectively controlled and the flow field structure can be optimized by adjusting the target load distribution reasonably. The limit of the maximum virtual displacement has a good effect on the stability of the calculation. The adjustment of the inlet and outlet geometry angle of stator blade has an important contribution to the improvement of aero-matching and calculation stability in the optimization process. At the design point, the flow rate of the Stage 35 compressor was basically unchanged, the isentropic efficiency was increased by $1.2 \%$, and total pressure ratio was also increased. The newly developed inverse method does not need modification of core flow solver, and has no requirement for grid structure. This makes it a potential to be applied to advanced solvers or commercial software.

\section{Declaration of Conflicting Interests}

The author(s) declared no potential conflicts of interest with respect to the research, authorship, and/or publication of this article.

\section{Funding}

The author(s) disclosed receipt of the following financial support for the research, authorship, and/or publication of this article: This work was supported by the National Key R\&D Plan of China (Grant No. 2017YFB0903605), The National Science Fund for Distinguished Young Scholars of China (Grant No. 51925604), The frontier science research project of CAS (Grant No. QYZDB-SSWJSC023), Transformational Technologies for Clean Energy and Demonstration, Strategic Priority Research Program of the Chinses Academy of Sciences (Grant No. XDA21070200) and Basic research plan of Guizhou Province, The Science and Technology Foundation of GuiThe Science and Technology Foundation of Guizhou Province (Grant No. [2019]1283).

\section{ORCID iDs}

Li Aiting (D) https://orcid.org/0000-0002-6104-7051 Wang Xing (D) https://orcid.org/0000-0002-4644-6599 Chen Haisheng (D) https://orcid.org/0000-0002-1383-9476

\section{References}

1. Lighthill M. A new method of two-dimensional aerodynamics design. ARC Technical Report No. 2112, 1945.

2. Hawthorne WR, Wang C, Tan CS, et al. Theory of blade design for large deflections-Part I: two-dimensional cascade. J Eng Gas Turbines Power 1984; 106: 346-353.

3. Borges JE. A three dimensional inverse method in turbomachinery - Part I: theory. ASME Paper No. GT 1989-136, 1989.

4. Zangeneh M. Inviscid-viscous interaction method for three-dimensional inverse design of centrifugal impellers. J Turbomach 1994; 116: 280-290.
5. Léonard $\mathrm{O}$ and Van Den Braembussche RA. Design method for subsonic and transonic cascade with prescribed Mach number distribution. ASME Paper No. GT 1991-18, 1991.

6. Demeulenaere A and Van Den Braembussche RA. Three-dimensional inverse method for turbomachinery blading design. ASME Paper No. GT 1996-39, 1996.

7. Dang $\mathrm{T}$ and Isgro V. Inverse method for turbomachine blades using existing time-marching techniques. ASME Paper No. GT 1994-20, 1994.

8. Dang T. Inverse method for turbomachine blades using shock-capturing techniques. AIAA Paper No. 19952465, 1995.

9. Dang T and Isgro V. Euler-based inverse method for turbomachine blades-Part I: two-dimensional cascades. AIAA J 1995; 33: 2309-2315.

10. Thompkins JWT and Tong SS. Inverse or design calculations for non-potential flow in turbomachinery blade passages. ASME Paper No. GT 1981-78, 1981.

11. Daneshkhah K and Ghaly WS. An inverse blade design method for subsonic and transonic viscous flow in compressors and turbines. Inverse Prob Sci Eng 2006; 14: 211-231.

12. Page JH, Hield $P$ and Tucker PG. Inverse design of $3 D$ multistage transonic fans at dual operating points. $J$ Turbomach 2013; 136: 041008.

13. Arbabi A, Ghaly W and Medd A. Aerodynamic inverse blade design of axial compressors in three-dimensional flow using a commercial CFD program. ASME Paper No. GT 2017-65194, 2017.

14. Nasrazadani SH, Nili-Ahmadabadi M and Noorsalehi MH. Upgrade and development of elastic surface inverse design method for axial compressor cascade with sharp-edged blades. Numer Heat Transfer, Part B 2019.

15. Yang J, Liu Y, Wang X, et al. An improved steady inverse method for turbomachinery aerodynamic design. Inverse Prob Sci Eng 2017; 25: 633-651.

16. Yang $\mathrm{C}, \mathrm{Wu} \mathrm{H}$, Liang $\mathrm{Y}$, et al. A novel threedimensional inverse method for axial compressor blade surface design. Arab J Sci Eng 2019; 44: 10169-10179.

17. Van Rooij MPC, Dang TQ and Larosiliere LM. Improving aerodynamic matching of axial compressor blading using a three-dimensional multistage inverse design method. $J$ Turbomach 2005; 129: 108-118.

18. Van Rooij MPC, Dang TQ and Larosiliere LM. Enhanced blade row matching capabilities via 3D multistage inverse design and pressure loading manager. ASME Paper No. GT 2008-50539, 2008.

19. Zhu Y, Ju Y and Zhang C. An experience-independent inverse design optimization method of compressor cascade airfoil. Proc IMechE, Part A: J Power and Energy 2019; 233: 431-442.

20. Liou M-S and Steffen CJ. A new flux splitting scheme. J Comput Phys 1993; 107: 23-39.

21. Yoon S and Jameson A. Lower-upper symmetricGauss-Seidel method for the Euler and Navier-Stokes equations. AIAA J 1988; 26: 1025-1026.

22. Spalart $\mathrm{P}$ and Allmaras S. A one-equation turbulence model for aerodynamic flows. AIAA Paper No. 19920439, 1992. 
23. Yang J, Liu Y, Wang X, et al. 3D viscous inverse design of turbomachinery using one-equation turbulence model. ASME Paper No. GT 2016-56332, 2016.

24. Ranjan Ray S and Zangeneh $\mathrm{M}$. A robust mixing plane and its application in three-dimensional inverse design of transonic turbine stages. J Turbomach 2014; 137: 011004 .
25. Reid L and Moore RD. Performance of single-stage axial-flow transonic compressor with rotor and stator aspect ratios of 1.19 and 1.26, respectively, and with design pressure ratio of 1.82. NASA Technical Paper No. $1338,1978$.

26. Numeca theory manual, Version 11.1. 